\title{
Translation and validation of the Revised Dental Beliefs Survey (DBS- R) in China
}

Patient perceptions of behaviours and attitudes of dentists are associated with dental fear and poor dental attendance in Western countries. However, there is a paucity of research exploring patient perceptions of the dentist in China. One reason for this may be the lack of a valid and reliable scale in Chinese (Standard Mandarin) to measure this. This study aimed to translate the Revised Dental Beliefs Survey (DBS-R) into Chinese and then explore the reliability and validity of this measure (both the short and longer versions) in a Chinese population. We translated the DBS-R using the forwardsbackwards method and pilot tested it on a small sample of adults in China. Following this, 480 Chinese adults completed the newly translated scale, as well as well as a standardised dental anxiety questionnaire (the Modified Dental Anxiety Scale Chinese version) to test convergent validity. 109 participants completed the DBS-R again 2 weeks later for test-retest reliability. Both versions of the Chinese DBS-R were internally consistent and demonstrated convergent validity; test-retest reliability was also good. Both versions of the scale performed similarly, but for now we would suggest the 28 -item version may be superior as items relating to the technical competence of the dentist appear important to Chinese adults.

Keywords: Dental beliefs - dental perceptions - dental anxiety - Chinese - scale validation.

Disclosure statement: No potential conflict of interest was reported by the authors 


\section{Introduction}

Negative perceptions of the dentist are associated with dental fear (Abrahamsson et al, 2006; Coolidge et al, 2005; 2010) and poor dental attendance in Western countries (Skaret et al, 2000). However, there is a paucity of research exploring views of the dentist in non-Western countries, such as China. Dental caries and periodontal disease are still highly prevalent in China and regular dental attendance is low (Chinese National Committee for Oral Health, 2018). However, China is experiencing rapid economic development and its dental health services are expanding with oral health highlighted as a priority (State Council of China, 2017). Within this, there is a commitment to investing in public oral health and moving towards prevention-centered practices (Zhou et al, 2018). Yet there is still limited data available on the factors responsible for uptake, of which negative perceptions of the dentist may be an explanatory variable. Lack of a valid and reliable scale in Chinese to measure perceptions and attitudes of the dentist may be one reason for the limited research in this area.

The Dental Beliefs Survey (DBS; Milgrom et al, 1985) and the revised version (DBS-R; Milgrom et al, 1995) were designed to assess the patient perceptions of the dentist. The 28 item DBS-R was developed in English and is divided into three subscales. Professionalism/Ethics relates to the technical competency and integrity of the dentist (e.g., 'I am concerned that dentists recommend work that is not really needed'). The Communication sub-scale encompasses items on the ease of dentistpatient communication and the attitude of the dentist (e.g., 'I'm concerned that dentists will embarrass me over the condition of my teeth'). The Lack of Control sub-scale relates to how much control the patient feels e.g., 'Once I am in the chair, I feel helpless'. Thus, it endeavors to assess the interpersonal relationship from the patient's 
perspective. It has been translated into several languages including Spanish (Coolidge et al, 2010), Swedish (Abrahamsson et al, 2006) and Turkish (Erciya et al, 2009).

Since the revised version was published, there have been studies which have explored its psychometric properties. Kvale et al (2004), in a study with fearful dental patients in the USA, found a modified factor solution of the DBS-R that encompassed Trust as a fourth factor (this mostly comprised of a subset of items from the Control subscale). The authors left out four of the items found not to contribute to the final model, thus the final four factor scale included 24/28 original DBS-R items. Further studies have evaluated the original and shorter DBS-R, with both measures indicating good validity and reliability across clinical and non-clinical samples (Coolidge et al, 2005; Abrahmsson et al, 2006). It has been suggested that the shorter version, may be of most use when researchers or clinicians have a specific interest in evaluating trust in the dentist (Coolidge et al, 2005).

To our knowledge there is no scale that exists in Chinese to measure views of the dentist. Such a scale would allow for assessment of dental attitudes and beliefs in a Chinese population and would also be useful for cross-cultural studies. Therefore, the aims of our study were to translate the DBS-R into Standard Mandarin and explore its reliability and validity (both long and short-form) in Chinese adults.

\section{Methods}

\section{Forward-Backwards Translation of the Revised Dental Beliefs Survey (DBS-R)}

A preliminary translated version of the DBS-R was obtained by following the forwardbackward method. The first step was to translate the DBS-R by the first author whose native language is Standard Mandarin (and is fluent in English) and who has in-depth experience of Chinese culture. To ensure the equivalence between the original and the translated forms, the back-translation method was used. Therefore, another bilingual 
individual (independent of the study), whose native language was Standard Mandarin, conducted a reverse translation. Both translators worked independently and the translator responsible for the back translation of the questionnaire did not have access to its original version. Once these translations were complete, a comparison between the original English version and the back-translated version was made by a panel comprising the original translator and two experts. Both experts were familiar with the DBS-R. After discussion, some minor modifications were made. This was then pilottested with a convenience sample of Chinese adults $(n=35)$ living in Tianjin, North China. The inclusion/exclusion criteria were that participants should be over 18 and have basic literacy/reading ability. The participants (Table 1) were asked to complete the scale and express their opinion on how easy/difficult it would be to understand. Seven out of thirty-five participants highlighted that they had never been a dental patient, and there were some items that pertained to direct experience e.g., 'Once I am in the chair I feel helpless'. Thus, we included an additional instruction at the beginning of the DBS-R for those who had never been a dental patient. We asked participants to score the questionnaire according to their expectations of the dentist/dental visit even if they had no direct experience. Participants agreed that they could easily rate the items based on expectations (including attending the dentist with others; seeing the dentist portrayed in the media and discussing dental treatment with family/friends). This instruction was translated into English and back into Chinese. This was checked again by the panel. No more modifications were made.

Insert Table 1 
We posted advertisements in a range of communities and colleges, and participants were given a small gift (a pen or a fan) for taking part. The study was approved by the Ethics Committee of Tianjin Normal University (reference APB20161205) and written informed consent was obtained.

\section{Procedure}

Participants were told that the study was exploring the different beliefs people have about going to the dentist. If they agreed to take part, they were given a questionnaire booklet (see Materials) to complete and return. Participants were asked to provide an email address if they were willing to complete the DBS-R again two weeks later (for test-retest reliability). From the 130 participants who provided their email addresses, 109 (35 males) completed the Chinese DBS-R (CDBS-R) a second time (43 from Tianjin city, 27 from Wuhan city and 39 from Dongying city).

\section{Materials}

A paper-based questionnaire booklet was devised for the study. It started with a request for demographics (e.g., gender, age and occupation). Brief questions about their dental attendance, treatment history and dental health behaviours (e.g., tooth-brushing) were also requested. Participants were then asked to complete our Chinese DBS-R (Appendix 1). Items were rated on a 5-point Likert-type scale, ranging from $1=$ not at all to 5=very much. Possible scores ranged from 28-140 for the longer and 24-114 for the shorter form. Higher scores indicate more negative perceptions/beliefs. Table 2 shows all items (in English) and items pertaining to each sub-scale. 
Finally, participants were asked to complete the Chinese Modified Dental Anxiety Scale (MDAS; Yuan et al, 2014), which is a dental anxiety measure originally developed in English (Humphris, Morrison \& Lindsay, 1995). It comprises five items related to dental experiences (e.g., "If you were about to have a tooth drilled, how you would you feel?") and is rated on a 5-point scale ranging from 'Not anxious' to 'Extremely anxious'. Overall scores range from 5-25 with a cut-off score of 19 to indicate a 'very dentally anxious' individual (King \& Humphris, 2010). The MDAS was included in order to test the convergent validity of the CDBS-R.

\section{Statistical Analyses}

In line with previous research (Abrahamsson et al, 2006; Coolidge et al, 2005) we analysed data for both the short and longer versions of the DBS-R for comparison purposes. We calculated Cronbach's Alpha to assess internal consistency, and testretest reliability was established using the intraclass correlation coefficient (ICC). Convergent validity was assessed by correlating the CDBS-R with the MDAS. We would expect a moderate correlation between dental beliefs and dental anxiety. In addition, employing a t-test we would expect there to be a significant difference in CDBS-R total scores between those scoring $>19$ and those scoring $<19$ on the MDAS. Moreover, we performed a t-test to confirm whether there was a difference in total CDBS-R scores between those who had never been to the dentist, and those who had. We would expect significantly higher scores for those who had never attended. To test whether there was a difference in dental beliefs across subscales, we calculated mean item totals and conducted a repeated measures ANOVA for both the three and four subscale versions. 


\section{Results}

There were 530 questionnaires distributed and 516 questionnaires returned. Of those returned, 36 had one or more items missing (across any of the questionnaires) so were removed from analysis. This left 480 completed questionnaires (325 females). Most participants were 20-30 years old and 71.9\% lived in urban areas. Twenty-two percent of the participants had never been to the dentist and tooth extraction was the procedure that most participants $(41 \%)$ had experienced. Twice-daily (or more often) toothbrushing was reported by majority of participants, though a third of participants reported less frequent brushing. For a full breakdown of demographics and the dental history and habits of the participants see Table 3.

\section{Insert Table 3}

The mean score was $85.2(\mathrm{SD}=17.6)$ for the 28 -item scale and $72.7(\mathrm{SD}=15.1)$ for the shorter form (Table 4). There was a significant difference in item mean scores across sub-scales on the 28 -item version $\mathrm{F}(2,474)=12.44 p<0.001)$. Post hoc tests showed that participants had significantly higher mean item totals on the Professionalism sub-scale than the Communication sub-scale $(p<0.005)$ and the Control sub-scale $(p<0.001)$. In addition, mean item totals were higher on the Control subscale than the Communication subscale $(p<0.05)$. On the 24 -item version there was also a significant difference in item mean scores $\mathrm{F}(3,474)=6.65 p<0.01)$ with participants having higher mean item totals on the Ethics sub-scale compared to the other three subscales; Communication $(p<0.01)$; Control $(p<0.05)$ and Trust $(p<0.001)$.

Insert Table 4 
As can be seen from Table 4 there was a high level of internal reliability for the CDBS-R long-form $(\alpha=0.94)$ and short-form $(\alpha=0.93)$. Sub-scales for both versions of the CDBS-R were also internally consistent though slightly lower values were noted on the shorter form. Test-retest data showed a strong ICC for both versions ( 0.94 for longer and 0.82 for shorter form).

\section{Insert Table 5}

The Modified Dental Anxiety Scale (MDAS) was internally consistent in our sample $(\alpha=0.85)$ and the mean anxiety score was $13.7(\mathrm{SD}=4.6)$. In order to assess validity we correlated the CDBS-R with the MDAS which showed a weak/moderate but significant correlation ( $r=0.38$ and $r=0.39, p<0.001$ for the longer and shorter versions).

As can be seen from Table 5 those who had never attended the dentist scored significantly higher on the CDBS-R overall and for each of the sub-scales across both versions of the scale. There were 75 participants $(15.6 \%)$ that had a very high anxiety score on the MDAS (score of 19 or above). Those in the highly anxious group, had significantly higher CDBS-R scores overall and for each of the sub-scales (Table 6).

\section{Insert Table 6}

\section{Discussion}

It is important to have a measure that can be used to reliably assess beliefs and attitudes about the dentist in China. Therefore, the aims of our study were to translate the DBS-R 
into Standard Mandarin and explore its reliability and validity (both long and shortform) in Chinese adults.

Across our sample, the psychometric properties for both the short and long version of the CDBS-R were found to be similar. Both versions had good reliability and validity. Internal consistency in both versions was high (0.93-0.94) with alpha levels similar to those found in studies with clinical and non-clinical samples (Abrahamsson et al, 2006; Coolidge et al, 2005; 2010). In addition, the subscales were all internally consistent. Test-retest data demonstrated that the CDBS-R is stable over a two-week period. Thus, our findings show the items on the CDBS-R measure a single underlying construct which is stable outside of the dental setting. This is particularly important considering the high number $(22 \%)$ of adults in our sample that are neverattenders.

The moderate but significant correlation between the Modified Dental Anxiety Scale (MDAS) and CDBS-R indicates that the two scales are tapping similar, but not identical, underlying constructs. For example, individuals could be highly fearful of certain dental procedures, but not necessarily have negative beliefs about the dentist. A similar finding was reported in the validation study for the Spanish version of the DBS$\mathrm{R}$ (Coolidge et al, 2010). Moreover, as an additional test of validity, when we divide the participants into high and low anxiety groups, we find that the highly anxious group have significantly higher CDBS-R scores.

The mean overall score for the CDBS-R appears high (85.2 shorter and 72.7 longer-form), especially when compared to studies conducted in Western countries. For 
example, Coolidge et al (2005), in their psychometric evaluation of both forms of the English version of the DBS-R, the mean score in their American student sample was 51.5 (longer-form) and 46.6 (shorter-form). Moreover, in a non-clinical study using the 28-item Spanish version of the DBS-R with a Spanish-speaking community sample of Hispanics in the USA, the mean was 61.2 (Coolidge et al, 2010). In addition, scores in our sample were high across the sub-scales on the CDBS-R (mean item scores 2.973.10). Abrahamsson et al (2006) compared item mean scores between students (1.72.3), general dental patients (1.4-1.8), periodontal patients (1.6-2.0) and fearful dental patients (2.7-3.6). Thus, our Chinese sample have beliefs most in line with fearful dental patients. Although a cross-cultural study would be valuable to explore this fully, our findings seem to indicate that beliefs about dentists in our Chinese sample may be more negative than found in some Western countries.

In our sample, there were differences across the sub-scales in terms of mean item total scores. For the 28-item version, participants had significantly higher mean item totals on the Professionalism/Ethics sub-scale than the Communication and the Control sub-scales. On the 24 -item version there was also a significant difference in item mean scores with participants having higher mean item totals on the Ethics subscale compared to the other three sub-scales. This would appear to indicate that Professionalism/Ethics of dentists are viewed most negatively by participants which has not been found previously in other studies. There are overlapping items in the Professionalism/Ethics and Ethics sub-scales across the two versions in that they both include items surrounding concerns about the dentist providing information, having the patient's best interests at heart and carrying out unnecessary work. Dental care is expensive across sectors in China; it may be that this contributes to a belief that dentists 
perform treatment that is expensive and not always necessary. However, this is speculative, and does not fully explain negative perceptions across other factors included in this dimension such as dentists withholding information. There is a need for further research to fully explore these beliefs.

Interestingly, Kvale et al (2004) in the 24-item DBS-R, omitted several items from the Professionalism/Ethics sub-scale that relate to the technical competence of the dentist. However, in our sample these items scored highly - Item 3 which is related to competency/skilled work was the highest scoring item. This may indicate two things. First, that technical competency and skill are important when viewing dentists in China (for those who have and have not been a dental patient), and as such should be explored further. Second, since only the 28-item CDBS-R includes these items, this may be the version of choice when assessing perceptions of the dentist in a non-clinical Chinese sample.

In our sample, there were low rates of regular dental attendance with $22 \%$ claiming they had never been a dental patient. Though this is based on self-report, it concurs with previous research exploring dental service utilization in China. Indeed, Zhu et al (2005) reported on the third oral health national survey within which $22 \%$ of respondents indicated they had never visited a dentist. Moreover, the fourth national survey (2015-2016) showed that only $20 \%$ of adults surveyed reported attending the dentist in the past year (Cheng et al, 2018). Within our pilot study, the high number of 'never attenders' (7/35) was also acknowledged. Therefore, we slightly modified the CDBS-R instructions to reflect this. We would argue that it is as important to assess the perceptions of those who have never attended, as it is to assess those who have. Indeed, 
perceptions of the behaviour and attitude of dentists could influence the decision to access dental care. When we compared total CDBS-R scores between those who had never attended the dentist and those who had - never-attenders had more negative perceptions of the dentist. This indicates there is a need to assess and address the negative perceptions of these non-attenders to hopefully help facilitate dental attendance.

There were strengths and limitations of our study. We followed a rigorous translation method (forwards-backwards method). We also piloted the measure and tried to ensure that the same meaning was conveyed on the translated measure while at the same time making it culturally meaningful. Although we sampled from three provinces, and aimed to get a varied sample in terms of demographics and experience, we cannot claim to generalise across China. In addition, there was an overrepresentation of females in our sample. However, it should be noted that across countries females do appear to participate in studies on dental-related beliefs and fear more often than males, across both clinical and non-clinical samples (Abrahmsson et al, 2006; van Wijk \& Hoogstraten 2003; 2005).

In conclusion, understanding cultural beliefs and attitudes towards dentists is important. The CDBS-R could be used to assess, and possibly provide a starting point, to help explore dental beliefs in the Chinese population. It would also be useful to facilitate cross-cultural comparative studies. Both versions of the scale performed similarly, but for now we would suggest the 28 -item version may be superior as items relating to the technical competence of the dentist appear important to Chinese adults that have and have not been dental patients. 


\section{References}

Abrahamsson KH, Hakeberg M, Stenman J, Öhrn K: (2006). Dental beliefs: evaluation of the Swedish version of the revised Dental Beliefs Survey in different patient groups and in a non-clinical student sample. Eur J Oral Sci 114:209-215.

Cheng Lm, Xu Rm, Xie YY, Gao XL, Wu HJ, Wang X, Feng XP, Tai BJ, Hu DY, Lin CH, Wang B, Zheng SG, Liu XN, Rong SW, Wang JW, Wang X, Si Y. (2018).

Utilisation of Oral Health Services and Economic Burden of Oral Diseases in China.

Chinese Journal of Dental Research 21: 4

Coolidge T, Heima M, Coldwell SE, Weinstein P, Milgrom P. (2005). Psychometric properties of the Revised Dental Beliefs Survey. Community Dent Oral Epidemiol 33:289-297.

Coolidge T, Hillstead MH, Farjo N, Weinstein P, Coldwell SE. (2010). Additional psychometric data for the Spanish Modified Dental Anxiety Scale, and psychometric data for a Spanish version of the Revised Dental Beliefs Survey. BMC Oral Health 10:12

Erciya K, Hamamci Z, Buyukozturk S, Ericya AF. (2009). Revised dental beliefs survey: reliability and validity of a 22-item modified Turkish version. Journal of Oral Rehabilitation 36: 831-839

Hu D, Hong X, Li X. (2011). Oral health in China - trends and challenges. International Journal of Oral Science $3: 7-12$

Humphris GM, Morrison T, Lindsay SJE. (1995). The modified dental anxiety scale: validation and United Kingdom norms. Community Dent Health 12: 143-150.

King K, Humphris G. (2010). Evidence to confirm the cut-off for screening dental phobia using the modified dental anxiety scale. Soc Sci Dent.1(1):21-28.

Kvale G, Milgrom P, Getz T, Weinstein P, Johnsen TB. (2004). Beliefs about professional ethics, dentist-patient communication, control and trust among fearful dental patients: the factor structure of the revised Dental Beliefs Survey. Acta Odontol Scand 62: 21-29.

Milgrom P, Weinstein P, Getz T. (1995). Treating Fearful Dental Patients: A Patient Management Handbook. 2nd edition. Seattle, WA: Continuing Dental Education, University of Washington.

Milgrom P, Weinstein P, Kleinknecht R, Getz T. (1985). Treating fearful dental patients: a clinical handbook. Reston, VA: Reston Publishing Company. 
National Committee for Oral Health (NCOH). (2018). Report of the Fourth National Oral Health Survey in China [In Chinese]. Beijing: People's Medical Publishing House.

Skaret E, Raadal M, Kvale G, Berg E. (2000). Factors related to missed and cancelled dental appointments among adolescents in Norway. Eur J Oral Sci 108: 175-183.

The State Council of China. (2017). The blueprint of health plan during the 13th fiveyear plan period. http://m.chem17.com/st286994/news_1074127.html

van Wijk AJ, Hoogstraten J. (2005). Experience with dental pain and fear of dental pain. J Dent Res 84: 947-950.

van Wijk AJ, Hoogstraten J. (2003). The Fear of Dental Pain questionnaire; construction and validity. Eur J Oral Sci 111: 12-18.

van Wijk, A.J., McNeil, D.W., Ho, C.J., Buchanan, H. and Hoogstraten, J. (2006). A short English version of the Fear of Dental Pain questionnaire. European Journal of Oral Sciences, 114: 204-208.

Yuan, S, Freeman, R, Lahti, S, Lloyd-Williams, F, Humphris, G. (2008). Some psychometric properties of the Chinese version of the modified dental anxiety scale with cross validation. Health Qual Life Outcomes 6:22.

Zhou X, Xu X, Li J, H T, Yin W, Fan Y, Zhang X. (2018). Oral health in China: From vision to action. International Journal of Oral Science 10: 1.

Zhu L, Petersen PE, Wang HY, Bian JY, Zhang BX (2005). Oral health knowledge, attitudes and behaviour of adults in China, International Dental Journal, 55, 231-241. 